\title{
On Partitions of a Rectangle into Rectangles with Restricted Number of Cross Sections
}

\author{
R. Ahlswede and A.A. Yudin*
}

\begin{abstract}
Consider partitions of a rectangle into rectangles with restricted number of cross sections. The problem has an information theoretic flavor (in the spirit of [3]): richness of two dimensional pictures under 1-dimensional restrictions of from local information to global information.

We present two approaches to upper bound the cardinality of such partitions: a combinatorial recursion and harmonic analysis.
\end{abstract}

\section{The Problem}

Given a partition of a rectangle into rectangles, such that any line parallel to a side of the initial rectangle intersects at most $n$ rectangles of the partition. The task is to estimate the maximal number of rectangles of the partition. We make now our concept of partition precise and consider a more general problem.

Definition 1. We say that a family of rectangles $\mathcal{S}$ gives a partition of the rectangle $F$, if

$$
\bigcup_{A \in \mathcal{S}} A=F
$$

and for $\forall A, B \in \mathcal{S}, A \neq B$ the intersection of $A$ and $B$ contains at most boundary points.

Definition 2. We say that the partition $\mathcal{S}_{F}$ has the property $(m, n)$, if any line parallel to the base of the rectangle $F$ intersects at most $m$ rectangles of the partition, and perpendicular to the base - at most $n$ rectangles.

The problem is to find

$$
f(m, n)=\sup \operatorname{card}\left(S_{F}\right)=\sup \left|\mathcal{S}_{F}\right|,
$$

where the supremum is taken over all partitions $\mathcal{S}_{F}$ with property $(m, n)$.

It is evident that $f(m, n)=f(n, m)$. In case $m=n$ one readily sees that

$$
f(n, n) \geq 2 f(n-1, n-1)+2 \geq 2^{n} \text { for } n \geq 2 .
$$

It seems natural to conjecture that the first inequality is actually an equality. By successively improving our methods we derive here a decreasing sequence of upper bounds on $f(m, n)$ and finally come close to the conjectured bound.

\footnotetext{
* Recently we learnt that a paper on such problems appeared already in 1993: D.J. Kleitman "Partitioning a rectangle into many sub-rectangles so that a line can meet only a few", DIMACS Series in Discrete Mathematics and Theoretical Computer Science, Volume 9, 95-107, 1993.
}

R. Ahlswede et al. (Eds.): Information Transfer and Combinatorics, LNCS 4123, pp. 941-954, 2006.

(C) Springer-Verlag Berlin Heidelberg 2006 


\section{A Crude Estimate}

Let us look at

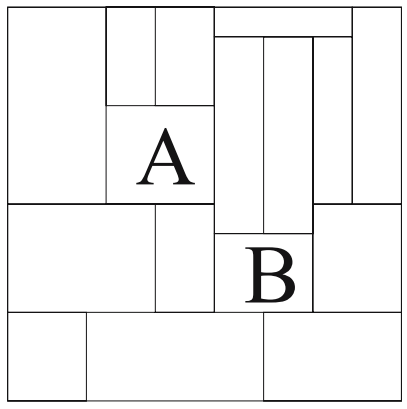

Fig. 1.

We associate with the partition $\mathcal{S}_{F}$ a graph $G_{\mathcal{S}}$. Every $A \in \mathcal{S}_{F}$ corresponds to a vertex of $G_{\mathcal{S}}$. For all $A, B \in \mathcal{S}_{F}, A \neq B$, there is an edge that connects them, iff $A$ and $B$ have common boundary, different from a point.

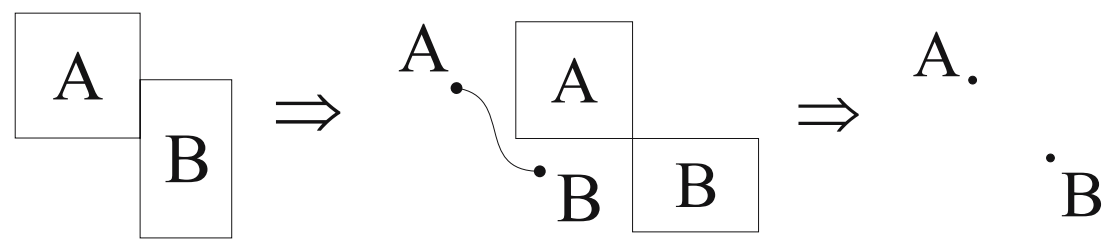

Fig. 2.

Fig. 3.

Now we state some properties of the graph $G_{\mathcal{S}}$.

1) We establish an upper bound for degrees of the vertices of this graph.

A vertex $A$ is incident to as many edges as the number of rectangles which have a common boundary with the rectangle $A$. It is evident that along any horizontal side there are at most $m$ of such rectangles, and along any vertical side there are at most $n$ of them, and thus around the rectangle $A$ there are at most $2(m+n)$ rectangles.

We have, therefore that for every vertex $v \in G_{\mathcal{S}}$

$$
\operatorname{deg} v \leq 2(m+n) .
$$

2) Now we find an upper bound for the diameter of the graph $G_{\mathcal{S}}$.

Given 2 vertices $A$ and $B$ (see Figure 4 ), let the vertex $X$ correspond to the rectangle that contains the point of intersection of the lines $\ell_{1}$ and $\ell_{2}$, $A \in \ell_{1}$ and $\ell_{1} \| \alpha \delta, B \in \ell_{2}$ and $\ell_{2} \| \gamma \delta$. 


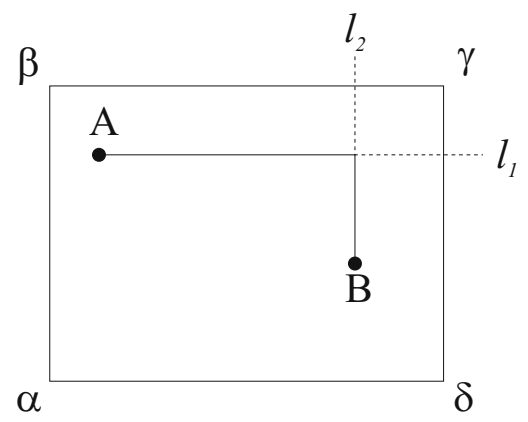

Fig. 4.

By definition, $\ell_{1}$ intersects at most $m$ rectangles of the partition, and $\ell_{2}$ intersects at most $n$ rectangles, hence the vertices $A$ and $X$ are connected by at most $m$ edges, and $X$ and $B$ by at most $n$ edges.

Therefore the diameter $d\left(G_{\mathcal{S}}\right) \leq m+n$. Thus we have

1) $\forall v \in G_{\mathcal{S}} \operatorname{deg} v \leq 2(m+n)$

2) $d\left(G_{\mathcal{S}}\right) \leq m+n$.

By inequality (1) on page 171 of $[1]$

$$
f(m, n) \leq 1+2(m+n) \frac{(2(m+n)-1)^{m+n}-1}{2(m+n)-2} \leq(2(m+n))^{m+n+1} .
$$

\section{A Sharper Estimate}

Choose on the side $\alpha \beta$ a rectangle $P$ with the biggest height $h$.

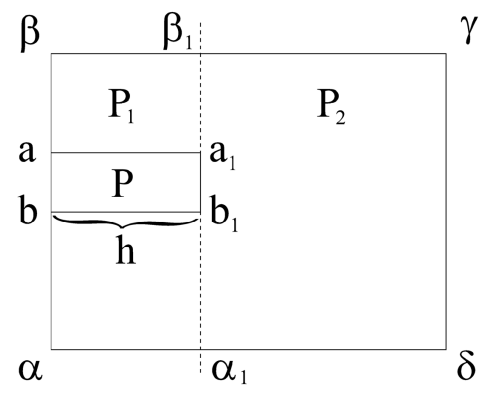

Fig. 5.

Then partition the rectangle $\alpha \beta \gamma \delta$ by line $\alpha_{1} \beta_{1}$ in two rectangles $P_{1}$ on the left side and $P_{2}$ on the right side of $\alpha_{1} \beta_{1}$. 


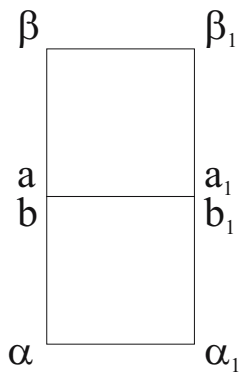

Fig. 6.

Adjust the rectangle $P_{1}$ by throwing away the rectangle $P$, and joining together the resulting two ones in the following way:

We get a new rectangle $P_{1}^{*}$.

Now we find the parameters $\left(m^{\prime}, n^{\prime}\right)$ of the rectangles $P_{1}^{*}$ and $P_{2}$.

For $P_{1}^{*}$ it is evident that $m^{\prime} \leq m$ and $n^{\prime} \leq n-1$. For $P_{2}$ it is evident that $m^{\prime} \leq m-1, n^{\prime} \leq n$.

Thus we get

$$
f(m, n) \leq 1+f(m, n-1)+f(m-1, n) .
$$

By adding 1 to both sides of this inequality and denoting $f(m, n)+1$ by $g(m, n)$, we get

$$
g(m, n) \leq g(m, n-1)+g(m-1, n) .
$$

By induction on $m+n$ we prove that

$$
g(m, n) \leq\left(\begin{array}{c}
m+n \\
n
\end{array}\right)
$$

Indeed,

$$
g(n, 1)=g(1, n)=n+1 \leq\left(\begin{array}{c}
1+n \\
n
\end{array}\right)
$$

and finally by (3.2) and the induction hypothesis

$$
g(m, n) \leq\left(\begin{array}{c}
m+n-1 \\
n-1
\end{array}\right)+\left(\begin{array}{c}
m+n-1 \\
n
\end{array}\right)=\left(\begin{array}{c}
m+n \\
n
\end{array}\right) .
$$

In the case $m=n$ we get

$$
f(n, n) \leq\left(\begin{array}{c}
2 n \\
n
\end{array}\right)-1 \sim c \frac{4^{n}}{\sqrt{n}} .
$$

\section{A Still Sharper Estimate}

The beginning is the same as in Section 3. Let us examine the estimate there more carefully. 


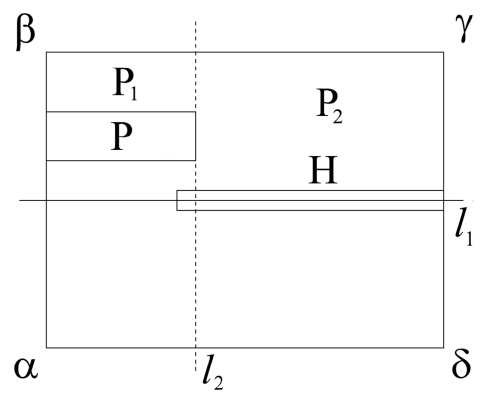

Fig. 7.

Let a horizontal line $\ell_{1}$ intersect $m$ rectangles of the rectangle $P_{1}$. Then there exists a rectangle $H$ of the partition from the side $\gamma \delta$ reaching the line $\ell_{2}$. The parameters of rectangles $P_{1}$ and $P_{2}$, after the reconstruction satisfy

$$
\begin{gathered}
m^{\prime}\left(P_{1}^{*}\right) \leq m \text { and } n^{\prime}\left(P_{1}^{*}\right) \leq n-1 \\
m^{\prime}\left(P_{2}^{*}\right) \leq m-s \text { and } n^{\prime}\left(P_{2}^{*}\right) \leq n-1 .
\end{gathered}
$$

So in this case the following inequality holds

$$
f(m, n) \leq f(m, n-1)+f(m-1, n-1)+1 .
$$

Again, by adding 1 to both sides of this inequality and denoting $f(m, n)+1$ by $g(m, n)$, we obtain

$$
g(m, n) \leq g(m, n-1)+g(m-1, n-1) .
$$

Thus if there exists a line $\ell_{1}$, which intersects $m$ rectangles of the rectangle $P_{1}$, then the inequality (4.1) holds.

Assume now that there is no such a line $\ell_{1}$, then the parameters $m^{\prime}$ and $n^{\prime}$ for $P_{1}^{*}$ and $P_{2}^{*}$ are

$$
\begin{gathered}
m^{\prime}\left(P_{1}^{*}\right) \leq m-1 \text { and } n^{\prime}\left(P_{1}^{*}\right) \leq n-1, \\
m^{\prime}\left(P_{2}^{*}\right) \leq m-1 \text { and } n^{\prime}\left(P_{2}^{*}\right) \leq n .
\end{gathered}
$$

Thus the following inequality holds

$$
g(m, n) \leq g(m-1, n-1)+g(m-1, n) .
$$

Combining (4.1) and (4.2) we get the following inequalities

$$
g(m, n) \leq\left\{\begin{array}{l}
g(m-1, n-1)+g(m-1, n) \\
g(m, n-1)+g(m-1, n-1)
\end{array}\right.
$$

We search for an estimate of the form

$$
g(m, n) \leq c \lambda^{(m+n)},
$$

where the constants $c$ and $\lambda$ are not known yet. We will determine them later. 
We get the estimate by induction. First let us determine $\lambda$. We have

$$
\begin{aligned}
g(m, n) & \leq \max \{g(m-1, n-1)+g(m-1, n), g(m, n-1)+g(m-1, n-1)\} \\
& \leq \max \left\{c \lambda^{m+n-2}+c \lambda^{m+n-1}, c \lambda^{m+n-1}+c \lambda^{m+n-2}\right\} \\
& \leq c\left(\lambda^{m+n-2}+\lambda^{m+n-1}\right)=c \lambda^{m+n}\left(\frac{1}{\lambda^{2}}+\frac{1}{\lambda}\right) .
\end{aligned}
$$

To be able to perform induction we need

$$
\frac{1}{\lambda^{2}}+\frac{1}{\lambda} \leq 1
$$

from which after calculations we find that we can set $\lambda_{0}=\frac{\sqrt{5}+1}{2}$.

The constant $c$ in (4.4) is determined from the initial step of the induction:

$$
g(1, n)=n+1 \leq c\left(\frac{\sqrt{5}+1}{2}\right)^{n+1}, \text { and thus } c=\sup _{n} \frac{n+1}{\left(\frac{\sqrt{5}+1}{2}\right)^{n^{2}}} \leq 1 .
$$

Then we get

$$
g(m, n) \leq\left(\frac{\sqrt{5}+1}{2}\right)^{m+n} .
$$

In the case $m=n$ this implies

$$
f(n, n) \leq\left(\frac{\sqrt{5}+1}{2}\right)^{2 n}-1<(1,62)^{2 n},
$$

which is of course better than $\frac{4^{n}}{\sqrt{n}}$ in (3.4), but much worse than the expected $2^{n}$.

\section{The Best Estimate Found so far by the Method of Recursion}

First we give some concepts.

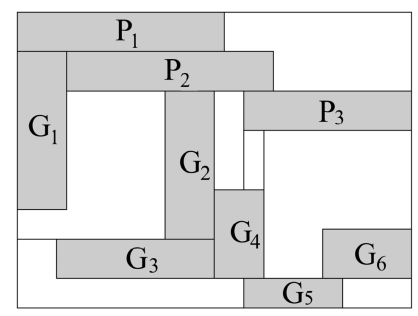

Fig. 8. 
Again, given a rectangle $F$ and a partition $\mathcal{S}$ of it, we call a sequence of rectangles $P_{1}, P_{2}, \ldots, P_{k}\left(P_{i} \neq P_{j}, i \neq j\right)$ a chain from the side $(\alpha \beta)$ to the side $(\gamma \delta)$ if any line $\ell \|(\alpha \beta)$ which intersects $F$, intersects at least one of $P_{1}, \ldots, P_{k}$.

Example: $P_{1}, P_{2}, P_{3}$ is a chain, $G_{1}, G_{2}, G_{3}, G_{4}, G_{5}, G_{6}$ is a chain.

We call a chain minimal if the number of rectangles in it is minimal. A minimal chain is extremal, if the sum of the lengths along the side $(\alpha \delta)$ of the rectangles of this chain is maximal (that is among all minimal chains we take the chain of maximum length).

It is straightforward to see that extremal chains exist.

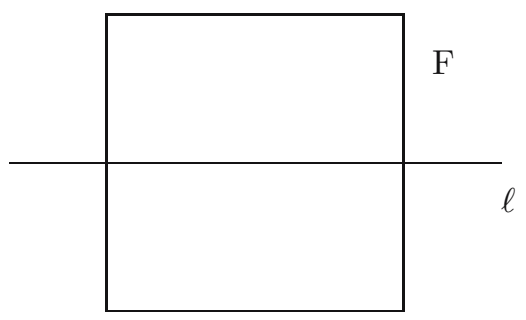

Fig. 9.

Indeed, as $\ell$ intersects no more than $m$ rectangles of $\mathcal{S}$, which evidently form a chain, there are chains. Since the cardinality of $S$ is a finite number, there is a chain with minimal number of elements, that is a minimal chain. Now the existence of extremal chains is evident, as the number of all chains, and so of minimal ones, is finite.

We classify now a partition $\mathcal{S}$ of the rectangle $F$ by the cardinality of its horizontal and vertical minimal chains.

We begin the consideration with the chains of cardinality $\geq 2$. Note that if there is a chain of cardinality one, then the partition $\mathcal{S}$ has the form

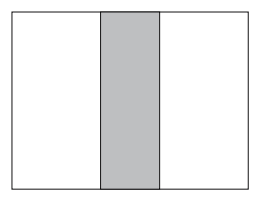

or

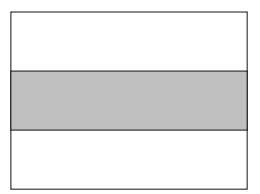

Fig. 10.

and this case is trivial to handle.

First we prove the following result.

Theorem 1. Let a partition $\mathcal{S}$ with parameters $m, n$ of a rectangle $F$ have an extremal chain of cardinality $\geq 5$. Then

$$
|\mathcal{S}| \leq 2 f(m-3, n-1)+f(m-2, n)+2 .
$$




\section{Proof}

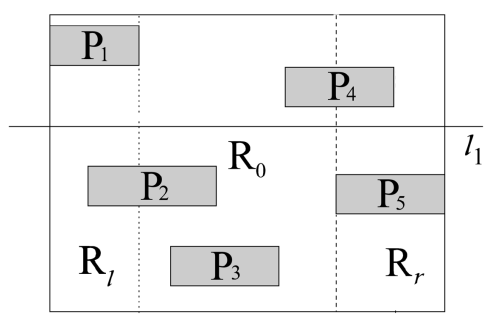

Fig. 11.

Without loss of generality let the rectangles $P_{1}, P_{2}, P_{3}, P_{4}$ and $P_{5}$ form an extremal chain. Partition the rectangle $F$ into three rectangles $R_{\ell}, R_{0}, R_{r}$ (as in Figure 11).

We estimate the parameters $m^{\prime}, n^{\prime}$ for the rectangles $R_{\ell}, R_{0}, R_{r}$. As after the adjustment the rectangle $P_{1}$ will be thrown away, we have

$$
n^{\prime} \text { for } R_{\ell} \leq n-1 \text {. }
$$

Now notice that $P_{3}, P_{4}, P_{5}$ cannot intersect $R_{\ell}$. Indeed, assume that $P_{3} \cap R_{\ell} \neq$ $\varnothing$. Then $P_{1}, P_{3}, P_{4}, P_{5}$ form a chain of cardinality 4 , which is a contradiction, since the chain $P_{1}, P_{2}, P_{3}, P_{4}, P_{5}$ is extremal. Similarly $P_{4} \cap R_{\ell}=R_{\ell} \cap P_{5}=\varnothing$.

Now we show that any line $\ell_{1}$ intersects at most $m-3$ rectangles of the partition $\mathcal{S}$, which belong to rectangle $R_{\ell}$. Assume to the opposite that $\ell_{1}$ intersects not less than $m-2$ rectangles of $R_{\ell}$. Denote by $Q_{1}$ the right most one of them. As there are no more than $m$ rectangles lying on $\ell_{1}$, there are at most 2 of them that do not intersect $R_{\ell}$. Denote them by $Q_{2}$ and $Q_{3}$. Again we come to a contradiction, since $P_{1}, Q_{1}, Q_{2}, Q_{3}$ form a chain, while $P_{1}, P_{2}, P_{3}, P_{4}, P_{5}$ is extremal. Thus we showed that

$$
m^{\prime} \text { for } R_{\ell} \leq m-3
$$

Similarly for $R_{r}$

$$
m^{\prime} \leq m-3 \text { and } n^{\prime} \leq n-1
$$

For $R_{0}$ evidently

$$
m^{\prime} \leq m-2 \text { and } n^{\prime} \leq n .
$$

Combining (5.1), (5.2), (5.3) and (5.4) we get the claim of Theorem 1.

Corollary. If any partition $\mathcal{S}$ with parameters $m$ and $n$ has an extremal chain of cardinality $\geq 5$, then

$$
f(m, n) \leq 2^{\frac{m+n}{2}} .
$$

Indeed, if the condition is fulfilled, then by Theorem 1

$$
g(m, n) \leq 2 g(m-3, n-1)+g(m-2, n),
$$

where $g(m, n)=f(m, n)+1$. Assuming, that the following estimate

$$
g(m, n) \leq c \cdot \lambda^{\frac{m+n}{2}}
$$


is correct for proper $c$ and $\lambda$, we get that $g(m, n) \leq 2 c \lambda^{\frac{m+n}{2}} \cdot \lambda^{-2}+c \lambda^{\frac{m+n}{2}} \cdot \lambda^{-1}=c \lambda^{\frac{m+n}{2}}\left(\frac{2}{\lambda^{2}}+\frac{1}{\lambda}\right)=c 2^{\frac{m+n}{2}}$, for $\lambda=2$.

As $f(1, n)=n$, the constant $c$ can be found from the condition

$$
1 \leq n \leq c \cdot 2^{\frac{n+1}{2}} \text { for all } n \in \mathbb{N} .
$$

It is evident that we can take $c=1$.

From now on only partitions with extremal chains of cardinality $\leq 4$ will be considered.

First we consider partitions $\mathcal{S}$ with chains of length 2 .

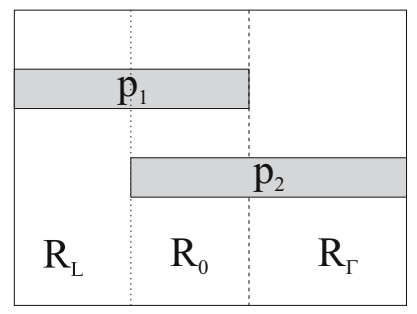

Fig. 12.

Now we find all possible collections of parameter values for rectangles $R_{\ell}, R_{0}, R_{r}$.

\begin{tabular}{c|c|c|c|l|l} 
& $R_{\ell}$ & $R_{r}$ & $R_{0}$ & Inequality & Solutions $\leq \sqrt{2}$ \\
\hline $\mathrm{N}$ & $m^{\prime}, n^{\prime}$ & $m^{\prime}, n^{\prime}$ & $m^{\prime}, n^{\prime}$ & & \\
\hline 1 & $m-1, n-3$ & $m-1, n-3$ & $m, n-2$ & $\frac{2}{\lambda^{4}}+\frac{1}{\lambda^{2}} \leq 1$ & yes \\
\hline 2 & $m-1, n-2$ & $m-1, n-3$ & $m, n-3$ & $\frac{2}{\lambda^{3}}+\frac{1}{\lambda^{4}} \leq 1$ & yes \\
\hline 3 & $m-1, n-3$ & $m-1, n-2$ & $m-1, n-2$ & $\frac{2}{\lambda^{3}}+\frac{1}{\lambda^{4}} \leq 1$ & yes \\
\hline 4 & $m-1, n-2$ & $m-1, n-2$ & $m-2, n-2$ & $\frac{2}{\lambda^{3}}+\frac{1}{\lambda^{4}} \leq 1$ & yes \\
\hline 5 & $m-1, n-3$ & $m-1, n-2$ & $m, n-3$ & $\frac{2}{\lambda^{3}}+\frac{1}{\lambda^{4}} \leq 1$ & yes \\
\hline 6 & $m-1, n-2$ & $m-1, n-2$ & $m-1, n-3$ & $\frac{2}{\lambda^{3}}+\frac{1}{\lambda^{4}} \leq 1$ & yes \\
\hline 7 & $m-1, n-3$ & $m-1, n-2$ & $m-1, n-3$ & $\frac{2}{\lambda^{3}}+\frac{1}{\lambda^{4}} \leq 1$ & yes \\
\hline 8 & $m-1, n-2$ & $m-1, n-1$ & $m-2, n-3$ & $\frac{1}{\lambda^{2}}+\frac{1}{\lambda^{3}}+\frac{1}{\lambda^{5}} \leq 1$ & no \\
\hline 9 & $m-1, n-2$ & $m-1, n-3$ & $m, n-3$ & $\frac{2}{\lambda^{3}}+\frac{1}{\lambda^{4}} \leq 1$ & yes \\
\hline 10 & $m-1, n-1$ & $m-1, n-3$ & $m-1, n-3$ & $\frac{1}{\lambda^{2}}+\frac{2}{\lambda^{4}} \leq 1$ & yes \\
\hline 11 & $m-1, n-2$ & $m-1, n-2$ & $m-1, n-3$ & $\frac{2}{\lambda^{3}}+\frac{1}{\lambda^{4}} \leq 1$ & yes \\
\hline 12 & $m-1, n-1$ & $m-1, n-2$ & $m-2, n-3$ & $\frac{1}{\lambda^{2}}+\frac{1}{\lambda^{3}}+\frac{1}{\lambda^{5}} \leq 1$ & no \\
\hline 13 & $m-1, n-2$ & $m-1, n-2$ & $m, n-4$ & $\frac{2}{\lambda^{3}}+\frac{1}{\lambda^{4}} \leq 1$ & yes \\
\hline 14 & $m-1, n-1$ & $m-1, n-2$ & $m-1, n-4$ & $\frac{1}{\lambda^{2}}+\frac{1}{\lambda^{3}}+\frac{1}{\lambda^{5}} \leq 1$ & no \\
\hline 15 & $m-1, n-2$ & $m-1, n-1$ & $m-1, n-4$ & $\frac{1}{\lambda^{3}}+\frac{1}{\lambda^{2}}+\frac{1}{\lambda^{5}} \leq 1$ & no \\
\hline 16 & $m-1, n-1$ & $m-1, n-1$ & $m-2, n-4$ & $\frac{2}{\lambda^{2}}+\frac{1}{\lambda^{6}} \leq 1$ & no \\
\hline
\end{tabular}

Here a few words about the table. The general procedure of finding an upper bound for $f(m, n)$ is the same as earlier: for each row of the values we get a 
recursion for $g(m, n)=f(m, n)+1$. Then we search for an upper bound of the form $g(m, n) \leq c \cdot \lambda^{m+n}$ with constants $c$ and $\lambda$ to be specified. The inequalities in the 5 -th column of the table guarantee that we can perform induction to prove the upper bound. So, their solutions can be specified as appropriate values for $\lambda$. The last column shows that in all cases (except those with entry "no", which are discussed below) one obtains the upper bound by setting $\lambda=\sqrt{2}$. The value of $c$ can be taken to be equal 1 .

As it can be seen from the table we get only two inequalities that give solutions $>\sqrt{2}$, namely

$$
\frac{1}{\lambda^{2}}+\frac{1}{\lambda^{3}}+\frac{1}{\lambda^{5}} \leq 1 \text { and } \frac{2}{\lambda^{2}}+\frac{1}{\lambda^{6}} \leq 1 .
$$

But in these cases we can put $\lambda=1.5$.

Now consider the case, when the partition $\mathcal{S}$ has an extremal chain of length $\geq 3$.

As there are just a few cases, we discuss them all. The following 4 cases are possible.

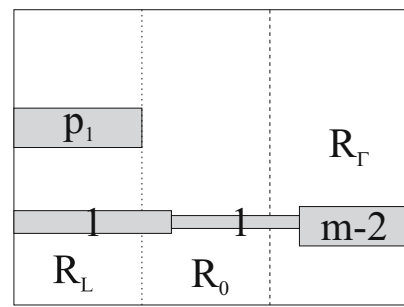

a)

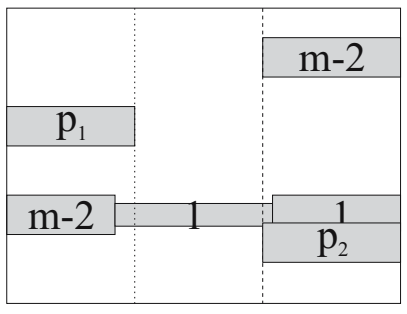

c)

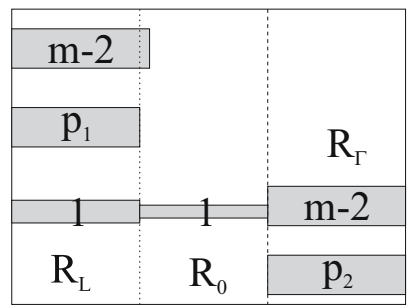

b)

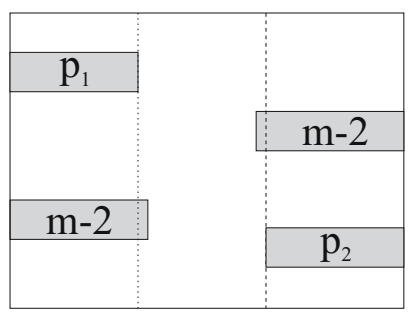

d)

Fig. 13.

In Figures a, b, c, d a rectangle with number $x$ inside means that a line intersecting it would intersect exactly $x$ rectangles of partition $\mathcal{S}$.

According to the four cases we obtain respectively:

a) $g(m, n) \leq g(m-1, n-1)+g(m-1, n-1)+g(m-2, n-2)$

b) $g(m, n) \leq g(m-2, n-1)+g(m-1, n-1)+g(m-2, n-1)$

c) $g(m, n) \leq g(m-1, n-1)+g(m-2, n-1)+g(m-2, n-1)$

d) $g(m, n) \leq g(m-2, n-1)+g(m-2, n-1)+g(m-2, n-1)$ 
The calculations show that the worst case is a). We get the inequality $\frac{2}{\lambda^{2}}+\frac{1}{\lambda^{4}} \leq$ 1 with solution $\lambda=\sqrt{\sqrt{2}+1}$.

Thus we proved

Theorem 2. For all $m, n \geq 1$ we have $f(m, n) \leq(\sqrt{2}+1)^{\frac{m+n}{2}}$. Thus $f(n, n) \leq$ $(2.414)^{n}$.

Remark 1. As it follows from Theorem 1, it is enough to examine partitions with extremal chains of cardinality $\leq 4$

2. So far we studied configurations only for chains in one dimension.

One can also consider pairs of chains. In that more general setting, the study of the following cases (see Figure 14) would yield sharper upper bounds for $f(m, n)$.

\begin{tabular}{c|c|c|c}
$\gamma\left(\ell_{2}\right) \backslash \gamma\left(\ell_{1}\right)$ & 2 & 3 & 4 \\
\hline 2 & $(2,2)$ & $(2,3)$ & $(2,4)$ \\
\hline 3 & & $(3,3)$ & $(3,4)$ \\
\hline 4 & & & $(4,4)$
\end{tabular}

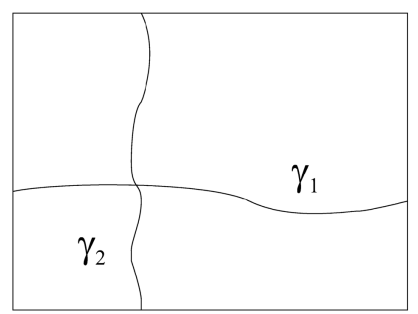

Let $\gamma_{1}$ and $\gamma_{2}$ be chains of cardinalities $\ell\left(\gamma_{1}\right)$ and $\ell\left(\gamma_{2}\right)$ resp.

Fig. 14.

Such an analysis, even for the case $(2,2)$, is rather complicated.

\section{Harmonic Analysis}

Given a partition $\mathcal{S}$ of a rectangle $F$.

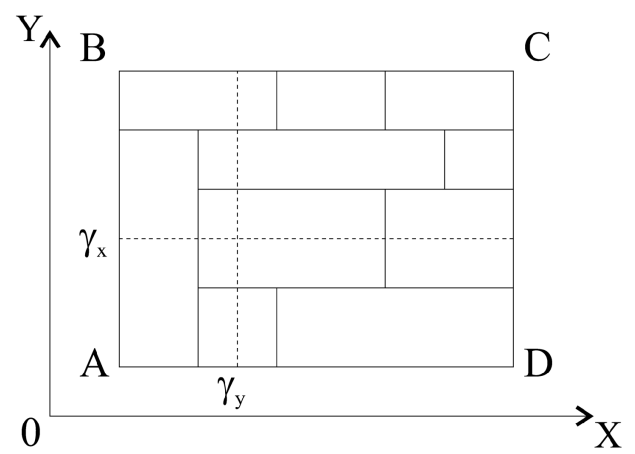

Fig. 15. 
We identify the opposite sides of the rectangle, so that $F$ becomes a torus and consider our problem on the torus $F$.

We have a partition $\mathcal{S}$ of the torus into rectangles such that any coordinate circle on $F$ intersects $\mathcal{S}$ in the following way:

Each $\gamma_{x}$ intersects at most $m$ rectangles and each $\gamma_{y}$ at most $n$ rectangles of the partition.

The problem is to estimate the number of rectangles in partition $\mathcal{S}$, that is $|\mathcal{S}|$.

Without loss of generality we may assume that $A B C D$ is a square with sides of length 1 .

Let $L_{y}(\varepsilon)$ be a strip of width $\varepsilon$ on the torus that is parallel to axis $O X$,
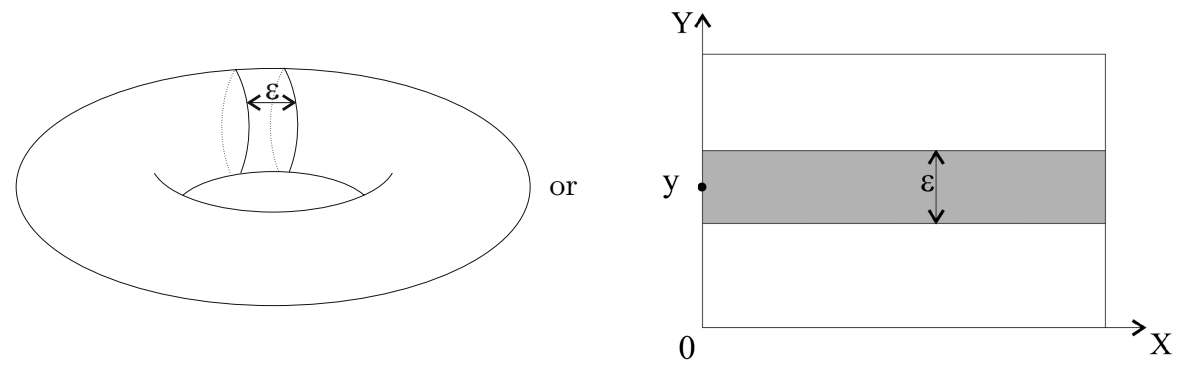

Fig. 16.

where $y$ is the $O Y$ coordinate of the center of that strip. Let

$$
\Phi_{\varepsilon}(y)=\sum_{P \in \mathcal{S} \text { and } P \cap L_{y}(\varepsilon) \neq \varnothing} 1
$$

that is $\Phi_{\varepsilon}(y)$ is equal to the number of rectangles of the partition $\mathcal{S}$, which have nonempty intersection with $L_{y}(\varepsilon)$.

Write the Fourier series for $\Phi_{\varepsilon}(y)$ :

$$
\Phi_{\varepsilon}(y)=c_{0}+\sum_{m: m \neq 0} c_{m} \ell(m, y)
$$

By the identity of Parseval we get

$$
\int_{0}^{1} \Phi_{\varepsilon}^{2}(y) d y=c_{0}^{2}+\sum_{m: m \neq 0} c_{m}^{2} \text { and thus } \int_{0}^{1} \Phi_{\varepsilon}^{2}(y) d y \geq c_{0}^{2}
$$

Now find $c_{0}$ :

$c_{0}=\int_{0}^{1}\left(\sum_{P \in \mathcal{S} \text { and } P \cap L_{y}(\varepsilon) \neq \varnothing} 1\right) d y=\sum_{P \in \mathcal{S}} \int_{\left\{y: P \cap L_{y}(\varepsilon) \neq \varnothing\right\}} d y=\sum_{P \in S}(\mu(P)+2 \varepsilon)=\sum_{P \in S} \mu(P)+2 \varepsilon|\mathcal{S}|$, 
where $\mu(P)$ is the length of the side of $P$ parallel to axis $O Y$. Let us now look at the integral $\int_{0}^{1} \Phi_{\varepsilon}(y) d y$ from another point of view. $\Phi_{\varepsilon}(y)$ is equal to the number of rectangles of the partition, which intersect the boundary of the strip $L_{y}(\varepsilon)$, evidently if there are no rectangles that are completely contained in $L_{y}(\varepsilon)$.

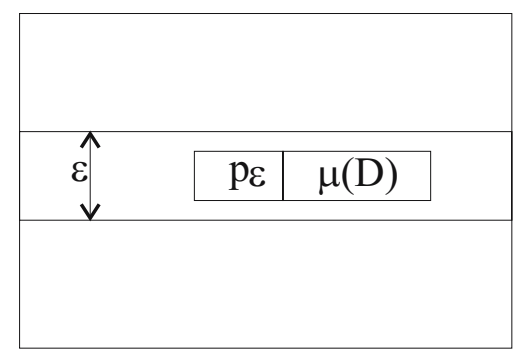

Fig. 17.

As the partition $\mathcal{S}$ has the property $(m, n)$, we can estimate $\Phi_{\varepsilon}(y) \leq 2 m$, if we don't take into consideration the rectangles, for which $\mu(P)<\varepsilon$.

By restricting ourselves to the set of rectangles $\mathcal{S}(\varepsilon)=\{P \in \mathcal{S}: \mu(P)>\varepsilon\}$, we obtain

$$
\left(\sum_{P \in \mathcal{S}(\varepsilon)} \mu(P)+2 \varepsilon|\mathcal{S}(\varepsilon)|\right) \cdot c_{0} \leq c_{0}^{2} \leq \int_{0}^{1} \Phi_{\varepsilon}^{2}(y) d y \leq \max _{y} \Phi_{\varepsilon}(y) \int_{0}^{1} \Phi_{\varepsilon}(y) d y \leq 2 m \cdot c_{0}
$$

and hence

$$
\sum_{P \in \mathcal{S}(\varepsilon)} \mu(P)+2 \varepsilon|\mathcal{S}(\varepsilon)| \leq 2 m
$$

Therefore

$$
2 \varepsilon|\mathcal{S}(\varepsilon)| \leq 2 m-\sum_{P \in \mathcal{S}(\varepsilon)} \mu(P) \text { implying }|\mathcal{S}(\varepsilon)| \leq \frac{m}{\varepsilon} .
$$

Thus we proved that the number of rectangles of the partition $\mathcal{S}$ with height $\geq \varepsilon$ does not exceed $\frac{m}{\varepsilon}$. Dealing similarly with the other dimension, we get that the number of rectangles of the partition $\mathcal{S}$ with length $\geq \varepsilon$ does not exceed $\frac{n}{\varepsilon}$. In summary

Theorem 3. The number of rectangles of partition $\mathcal{S}$ with exception of those, both sides of which have length less than $\varepsilon$, does not exceed

$$
\frac{m}{\varepsilon}+\frac{n}{\varepsilon}=\frac{m+n}{\varepsilon} \text {. }
$$

Corollary. Given a square $K_{n}$ on checked paper with the length of side $2^{n}$, which is partitioned into rectangles (that consist of entire checks), so that any 
line parallel to a side of the square intersects at most $n$ rectangles, then the number of rectangles of the partition is $\leq 2 n \cdot 2^{n}$.

Proof: Apply to $K_{n}$ homothety with coefficient $2^{-n}$, and take in the previous claim

$$
\varepsilon=2^{-n} \text {. }
$$

Then we get that the number of rectangles of the partition which have at least one dimension not less than $\varepsilon=2^{-n}$ (and in the given case they all do satisfy this condition) is

$$
f(n, n) \leq \frac{n+n}{2^{-n}}=2 n \cdot 2^{N} .
$$

Recall that the lower bound for $f(n, n)$, which is conjectured to be tight, is $2^{n}$.

Remark: The main difficulty for the analytic approach to this problem is the tight interlacing of combinatorial and metrical — old structures of the partition.

It seems, that if there are "small" rectangles in the structure of the partition, then the partition can be adjusted so that these small rectangles are removed, while preserving the combinatorial structure. But we don't know yet how to do it.

\section{References}

1. B. Bollobás, Extremal Graph Theory, Academic Press, London, 1978.

2. J. Bourgain, Harmonic analysis and combinatorics: how much may they contribute to each other? Published in Mathematics: Frontiers and Perspectives, V. Arnold, M. Atiyah, P. Lax, and B. Mazur, Editors, 13-32, 2000.

3. R. Ahlswede, Coloring hypergraphs: A new approach to multi-user source coding, Part I, Journ. of Combinatorics, Information and System Sciences, Vol. 4, No. 1, 76-115, 1979; Part II, Vol. 5, No. 3, 220-268, 1980. 